\title{
Caroli's Disease Associated to Renal Cysts: Case Report and Review of the Literature
}

\author{
F. Haddar ${ }^{1 *}$, F. Bellouhou ${ }^{1}$, A. Ait Errami ${ }^{1}$, S. Oubaha ${ }^{2}$, Z. Samlani ${ }^{1}$, K. Krati ${ }^{1}$
}

${ }^{1}$ Department of Gastroenterology, Mohammed VI University Hospital, Marrakech, Morocco

${ }^{2}$ Department of Physiology, Faculty of Medicine and Pharmacy at Cadi Ayyad University, Marrakech, Morocco

DOI: $10.36347 /$ simcr.2020.v08i02.017

| Received: 03.02.2020 | Accepted: 10.02.2020 | Published: 14.02.2020

*Corresponding author: F. Haddar

Abstract

Caroli's disease is characterized by multifocal segmental dilatation of the intrahepatic bile ducts. It is rare congenital condiction, which appears to autosomal recessively inherited in most cases. There are two forms of disease, one associated with congenital hepatic fibrosis and simple form occurring alone. Recent reports suggest that simple form may be as common as thar with congenital hepatic fibrosis. Other conditions, including choledochal cyst and renal cystic disease, are frequently associated. The major clinical feature is reccurent cholangitis, which may be complicated by intrahepatic calculi and hepatic abscess formation. There is good evidence that malignancy complicates Caroli's disease in approximately $7 \%$ of cases. The diagnosis rests on demonstrating that the cystic liver lesions are in continuity with the biliary tree. Modern imaging techniques allow the diagnosis to be made more easily and without invasive imaging of the biliary tree. The treatment depends on the clinical features and the location of the biliary abnormality. When the disease is localized to one hepatic lobe, hepatectomy relieves symptoms and appears to remove the risk malignancy. The patients with bilobar disease not amenable for liver transplantation but operated with Fag Kan intervention can be managed not only with removal of bile ducts stones when symptomatic, but with insertion of multiple plastic stents, that must be changed periodically, for example every three months during a daily hospital access.

Keywords: Adult polycystic, kidney disease, caroli's disease.

Copyright @ 2020: This is an open-access article distributed under the terms of the Creative Commons Attribution license which permits unrestricted use, distribution, and reproduction in any medium for non-commercial use (NonCommercial, or CC-BY-NC) provided the original author and source are credited.

Abbreviations: APKD, Adult Polycystic Kidney Disease; ERCP, Endoscopic Retrograde Cholangiopancreatography; MRCP: Magnetic Resonance Cholangio Pancreatography.

\section{INTRODUCTION}

Caroli's disease or communicating cavernous ectasia of the intrahepatic bile ducts is a rare autosomal recessive disorder which occurs due to the abnormal development of the intrahepatic bile ducts secondary to intrauterine vascular insult [1]. It is characterized by non-obstructive dilatation of the intrahepatic biliary radicles. Caroli's disease may be diffuse or may be localized to a lobe or a segment of the liver, usually the left [2]. The disease is recognized in its two formssimple (classic); in which the intrahepatic biliary duct dilatation is seen without hepatic fibrosis and Caroli's syndrome (fibrous); in which there is hepatic fibrosis in addition that may be accompanied by the cascade of events that follow portal hypertension. In contrast enhanced computed tomography (CECT), the 'central dot sign' representing the vascular bundle in dilated biliary duct is pathognomonic. Early recognition of the disease and its complications is required for timely management of the patient.

\section{CASE REPORT}

A 59-year-old female presented with intermittent right upper quadrant pain for the past five months. There was no history of fever or jaundice. The blood tests were unremarkable except for slightly raised levels of SGOT and SGPT. The general physical examination was also normal with no hepatomegaly or splenomegaly. On ultrasound of the abdomen, multiple tortuous tubular anechoic structures were seen in the right lobe of the liver. These were communicating with the right biliary tree. On Doppler imaging, the right portal vein branches were accompanying these dilated channels and some of these dilated channels were also showing traversing septae. The left biliary ducts, common hepatic duct, common bile duct and gall 
bladder were normal. Contrast enhanced CT abdomen showed saccular dilatation of biliary radicals which were confined to the right lobe (Fig-1). A small enhancing dot representing portal vein branch was seen in the center of some of these cystic dilatations giving the typical 'central dot' sign. The left and extrahepatic biliary tree and the gall bladder were normal. Both the kidneys showed multiple small simple cysts (Fig-2). There was no evidence of hepatic mass or portal hypertension on both Doppler and CT imaging. Esophageal varices, splenomegaly or ascites were not seen. The radiographic imaging was consistent with simple Caroli's disease in localized form. Because of increased risk of cholangiocarcinoma, the patient was offered hepatic lobectomy as the treatment which the patient had denied at present. So the patient is managed with analgesics along with close follow up for the possibilities of complications.

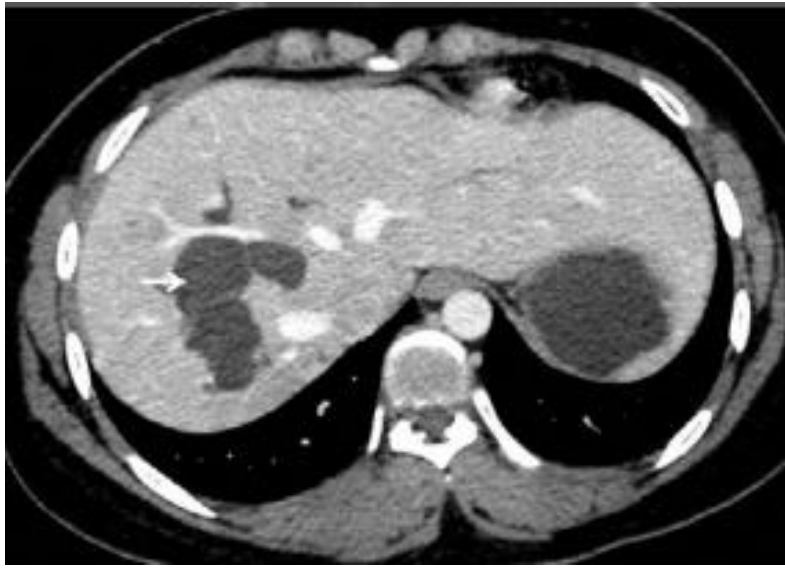

Fig-1: Contrast enhanced CT images (axial) show hypoechoic saccular dilatations in right lobe of the liver communicating with right hepatic duct consistent with dilated biliary radicles (white arrow)
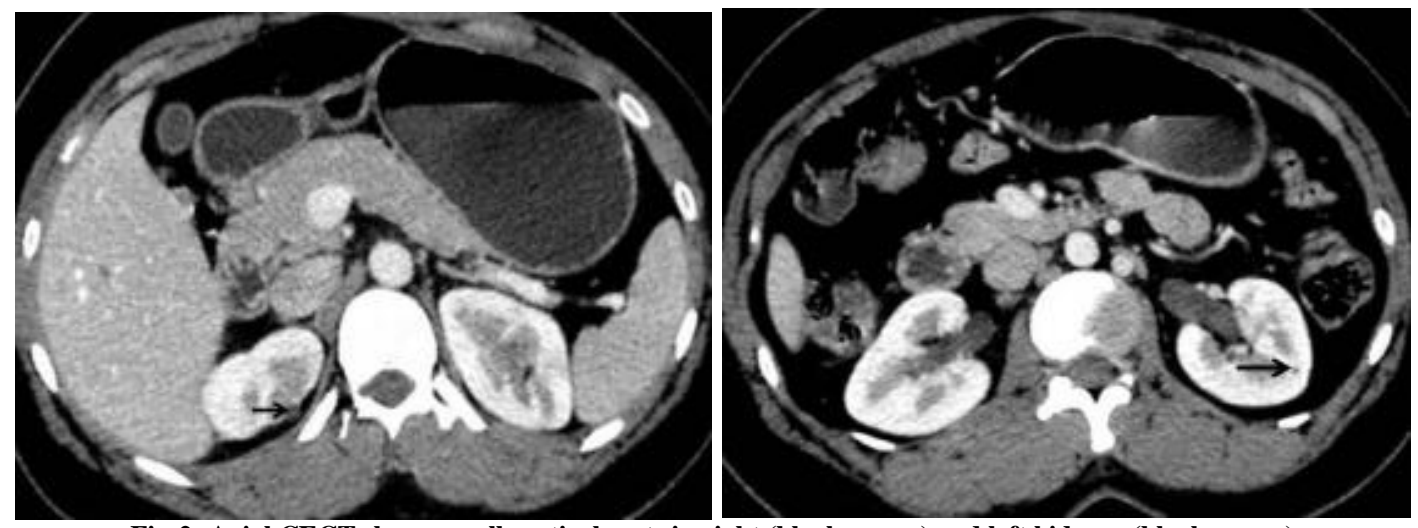

Fig-2: Axial CECT shows small cortical cysts in right (black arrow) and left kidneys (black arrow)

\section{DISCUSSION}

Caroli's disease is a rare congenital disorder characterized by focal or diffuse saccular dilatation of intrahepatic biliary radicals. It was first described by Caroli et al., in 1958 [3]. It is also classified as type V choledochal cyst [4]. The estimated incidence is 1 in $1,000,000$ population. Most cases follow autosomal recessive inheritance. There is no sex predilection [5]. The exact pathogenesis is unclear but it is suggested that a prenatal vascular accident causing occlusion of the hepatic artery is the triggering factor [6]. This compromises biliary ductal circulation and leads to complete or partial derangement in the normal remodeling of the ductal plate which is a layer of hepatic precursor cells that surrounds the portal vein branches and is the anlage of intrahepatic bile ducts. Varying severity of destructive inflammation and segmental dilatation of the bile ducts follow [7].

It should be remembered that the remodeling of the hepatic ductal plates begins in utero from the hilum to the periphery of the liver with successive development of the larger ducts first, then the segmental ducts, the interlobular ducts and finally the smallest bile ductules [1]. If the biliary malformation involves the ductal plate of the large central intrahepatic ducts only, it causes the simple type of Caroli's disease. If the ductal plates of both the central and the smaller peripheral intrahepatic ducts are malformed, Caroli's disease with congenital hepatic fibrosis results known as Caroli's syndrome [1].

Although the disorder is congenital, the patient is asymptomatic in first two decades of life and may remain so for the entire life. Intermittent abdominal pain is the most common symptom. Other presenting features are fever and jaundice. The patients with Caroli's disease and congenital hepatic fibrosis may reveal features of portal hypertension like ascites and esophageal variceal hemorrhage [5]. The significance of recognizing Caroli's disease as a cause of biliary stasis is its frequent association with various complications. The common ones are stone formation and cholangitis. Bile duct dilatation leads to bile stagnation and then to lithiasis. Recurrent cholangitis is the primary cause of morbidity and mortality. Less frequent complication is cholangiocarcinoma with an incidence of 7-14\% [8]. Our patient had come with intermittent abdominal pain and the disease was recognized before the development of any complication while the most common mode of presentation is acute cholangitis seen in $64 \%$ of cases [5]. 
Non invasive imaging techniques like ultrasound, CT and MRCP help in the diagnosis. Therefore invasive procedures like ERCP and PTC are usually not required. On sonography, the dilated biliary channels are anechoic and on CT are hypodense. The diagnosis rests on demonstration of continuity of these saccular dilatations with the biliary tree. The fibrovascular bundle which comprises of portal vein radical and branch of hepatic artery can be seen traversing through these biliary dilatations as enhacing septae or central dot giving the 'central dot' sign which can be seen on both contrast enhanced CT and Doppler. MRI provides better information regarding location, severity and extent of the disease. However the histopathological examination of the resected specimen is the gold standard. The differentials to be considered are primary sclerosing cholangitis (PSC), recurrent pyogenic cholangitis, polycystic liver disease, biliary papillomatosis and sometimes obstructive dilatation [5]. Biliary ductal dilatation, stenosis, intrahepatic calculi and malignancy may be associated with Caroli's disease as well as with PSC, however, in PSC the dilatation is rarely saccular and is typically more isolated and fusiform which is not the characteristic of Caroli's disease. All these biliary changes are also seen with recurrent pyogenic cholangitis and this differential is difficult to exclude on imaging alone. However, in recurrent pyogenic cholangitis the patient is septic while it is not so in simple Caroli's disease. In polycystic hepatic disease, the cysts do not communicate with bile ducts [8]. MRI is superior to CT for differentiating these conditions. Various renal disorders like autosomal dominant polycystic kidney disease, autosomal recessive polycystic kidney disease, medullary sponge kidney and medullary cystic disease may be associated [1].

Medical treatment of CS includes antibiotics for cholangitis, ursodeoxycholic acid for cholestasis, beta blocker therapy and treatment of varices for portal hypertension [5, 8, 9]. Surgical treatment depends on the clinical features and the location of the biliary abnormalities. Seg-mental or lobar hepatic resection provides symptomatic relief and removes the risk of malignancy when the abnormalities are localized. In case of diffuse involvement of liver, treatment options include conservative management, endoscopic therapy (sphincterotomy for clearance of intra-hepatic stone), internal biliary bypass procedures and liver transplantation [5, 8, 9-11]. If concomitant renal failure ensues from the dysplastic kidneys, liver transplantation combined with renal transplantation might be warranted [12]. Genetic counseling and screening of family members is important as the disease has an autosomal recessive inheritance.

\section{CONCLUSION}

Caroli's disease is a rare but important cause of biliary stasis. The patient should be offered appropriate surgical intervention otherwise close follow up is mandatory for the possible development of the complications.

Conflict of Interest: There is no conflict of interest to disclose.

\section{REFERENCES}

1. Levy AD, Rohrmann Jr CA, Murakata LA, Lonergan GJ. Caroli's disease: radiologic spectrum with pathologic correlation. American Journal of Roentgenology. 2002 Oct;179(4):1053-7.

2. Jarry J, Saric J. Monolobar Caroli's disease. HPB. 2009 Jun 1;11(4):371.

3. Patel S, Kulkarni A, Agrawal R, Nathan G. Monolobar caroli's disease: a case report. The American Journal of Gastroenterology. 2003 Sep 1;98(s9):205-206.

4. Todani T, Watanabe Y, Narusue M, Tabuchi K, Okajima K. Congenital bile duct cysts: classification, operative procedures, and review of thirty-seven cases including cancer arising from choledochal cyst. Am Journal Surgery, 1977;134(2):263-9.

5. Yonem O, Bayraktar Y. Clinical characteristics of Caroli's disease. World journal of gastroenterology: WJG. 2007 Apr 7;13(13):19301933.

6. Sato Y, Ren XS, Nakanuma Y. Caroli's disease: current knowledge of its biliary pathogenesis obtained from an orthologous rat model. International journal of hepatology. $2011 \mathrm{Jul}$ 6;2012.

7. Fulcher AS, Turner MA, Sanyal AJ. Case 38: Caroli disease and renal tubular ectasia. Radiology. 2001 Sep;220(3):720-3.

8. Bavikar R, Kulkarni R. Caroli's syndrome: A case report. Curr Pediatr Res, 2011;15:59-60.

9. Karim AS. Caroli's disease. Indian Pediatr, 2004;41:848-50.

10. Gunay-Aygun M. Liver and kidney disease in ciliopathies. Am J Med Genet C Semin Med Genet, 2009;151C:296-306.

11. Mrowka C, Adam G, Sieberth HG, Matern S. Caroli's syndrome associated with medullary sponge kidney and nephrocalcinosis. Nephrol Dial Transplant, 1996;11:1142-5.

12. Kim JT, Hur YJ, Park JM, Kim MJ, Park YN, Lee JS. Caroli's syndrome with autosomal recessive polycystic kidney disease in a two month old infant. Yonsei Med Journal, 2006;47:131-4. 